\title{
Analysis, Design and Empirical Validation of a Smart Campus Based on LoRaWAN ${ }^{\dagger}$
}

\author{
Paula Fraga-Lamas ${ }^{1, *}$, Mikel Celaya-Echarri ${ }^{2}$, Peio Lopez-Iturri ${ }^{3}$, \\ Tiago M. Fernández-Caramés ${ }^{1, *}$, Leyre Azpilicueta ${ }^{2}$, Erik Aguirre ${ }^{3}$, Manuel Suárez-Albela ${ }^{1}$, \\ Francisco Falcone ${ }^{3}$ and Luis Castedo ${ }^{1}$ \\ 1 Department of Computer Engineering, Faculty of Computer Science, Universidade da Coruña, \\ 15071 A Coruña, Spain; m.albela@udc.es (M.S.-A.); luis@udc.es (L.C.) \\ 2 School of Engineering and Sciences, Tecnológico de Monterrey, 64849 Monterrey, Mexico; \\ mikelcelaya@gmail.com (M.C.-E.); leyre.azpilicueta@itesm.mx (L.A.) \\ 3 Department of Electric, Electronic and Communication Engineering, Public University of Navarre, \\ 31006 Pamplona, Spain; peio.lopez@unavarra.es (P.L.-I.); erik.aguirre@unavarra.es (E.A.); \\ francisco.falcone@unavarra.es (F.F.) \\ * Correspondence: paula.fraga@udc.es (P.F.-L.); tiago.fernandez@udc.es (T.M.F.-C.); \\ Tel.: +34-981167000 (ext. 6051) (P.F.-L.) \\ + Presented at the 5th International Electronic Conference on Sensors and Applications, 15-30 November 2018; \\ Available online: https:/ / ecsa-5.sciforum.net/.
}

Published: 14 November 2018

\begin{abstract}
Internet of Things (IoT) applications for smart environments demand challenging requirements for wireless networks in terms of security, coverage, availability, power consumption, and scalability. The technologies employed so far to cope with IoT scenarios are not yet able to manage simultaneously all these demanding requirements, but recent solutions like Low-Power Wide Area Networks (LPWANs) have emerged as a promising alternative to provide low-cost and low-power consumption connectivity to nodes spread throughout a wide area. Specifically, the Long-Range Wide Area Network (LoRaWAN) standard is one of the most recent developments, receiving attention from both industry and academia. This work presents a comprehensive case study on the use of LoRaWAN under a realistic scenario within a smart city: a smart campus. Such a medium-scale scenario has been implemented through an in-house-developed 3D ray launching radio planning simulator that takes into consideration traffic lights, vehicles, people, buildings, urban fixtures, and vegetation. The developed tool is able to provide accurate radio propagation estimations within the smart campus scenario in terms of coverage, capacity, and energy efficiency of the network. These results are compared with an empirical validation in order to assess the operating conditions and the system accuracy. Moreover, the presented results provide some guidelines for IoT vendors, network operators, and city planners to investigate further deployments of LoRaWAN for other medium-scale smart city applications.
\end{abstract}

Keywords: IoT; smart campus; LP-WAN; LoRaWAN; 3D ray launching; smart cities; Wireless Sensor Networks (WSN)

\section{Introduction}

A smart campus deploys smart city technologies and Internet of Things (IoT) solutions [1,2] to connect, monitor, control, optimize, and automate the campus systems. Potential applications are able to provide energy savings, waste reduction, or more efficient, context-aware, and sustainable buildings and transportation infrastructure. In this scenario, Low-Power Wide Area Network (LPWAN) technology has emerged as an enabling technology for IoT and Machine-to-Machine (M2M) 
communications [3], mainly due to its features regarding range, cost, power consumption, and capacity. Recently, numerous platforms have arisen following this paradigm, like SigFox, Ingenu, Weightless, or LoRaWAN [4]. In the case of LoRaWAN, it is gaining momentum from both industry and academia. LoRaWAN defines the communication protocol and the system architecture for the network; it uses LoRa as the physical layer, which is able to create long-range communication links and makes use of a Chirp Spread Spectrum (CSS) modulation that conserves the power features of Frequency-Shift Keying (FSK) while increasing its communication range capabilities.

Smart city technologies have been extensively researched in the literature, regarding the specific use of LPWAN technologies. However, although there are several recent studies on the performance of LoRa/LoRaWAN technology for certain scenarios, only a few describe real-world LoRaWAN deployments explicitly aimed at providing communications to a smart campus. For instance, Loriot et al. [5] conducted LoRaWAN measurements on a French campus both outdoors and indoors and showed that the technology is able to provide good performance over the major part of the campus. Another development is presented in [6], where the authors set up a LoRaWAN-based air quality system on their campus. Another interesting paper is [7], which details the design of a LoRa mesh network system within a campus.

In contrast to the articles previously mentioned, in this paper is presented a smart campus scenario that has been implemented through an in-house-developed 3D ray launching radio planning simulator. The results obtained by such a simulator are validated by first comparing them with empirical LoRaWAN measurements and then analyzing the performance of LoRaWAN on the campus.

\section{Designing a Smart Campus}

This article presents a case study conducted in the northwest of Spain on the Campus of Elviña of the University of A Coruña. The campus covers an area of $26,000 \mathrm{~m}^{2}$, and it includes elements typically found in an urban environment. Figure 1 shows an aerial view of the campus. It must be noted that two rectangular scenarios were selected within the campus (red on the left and green on the right) to cover eight faculties. The rectangular shape is required by the 3D ray launching simulator. The points marked as $M, A, Q$, and $R$ correspond to different reception distances from the transmitter $(T)$.

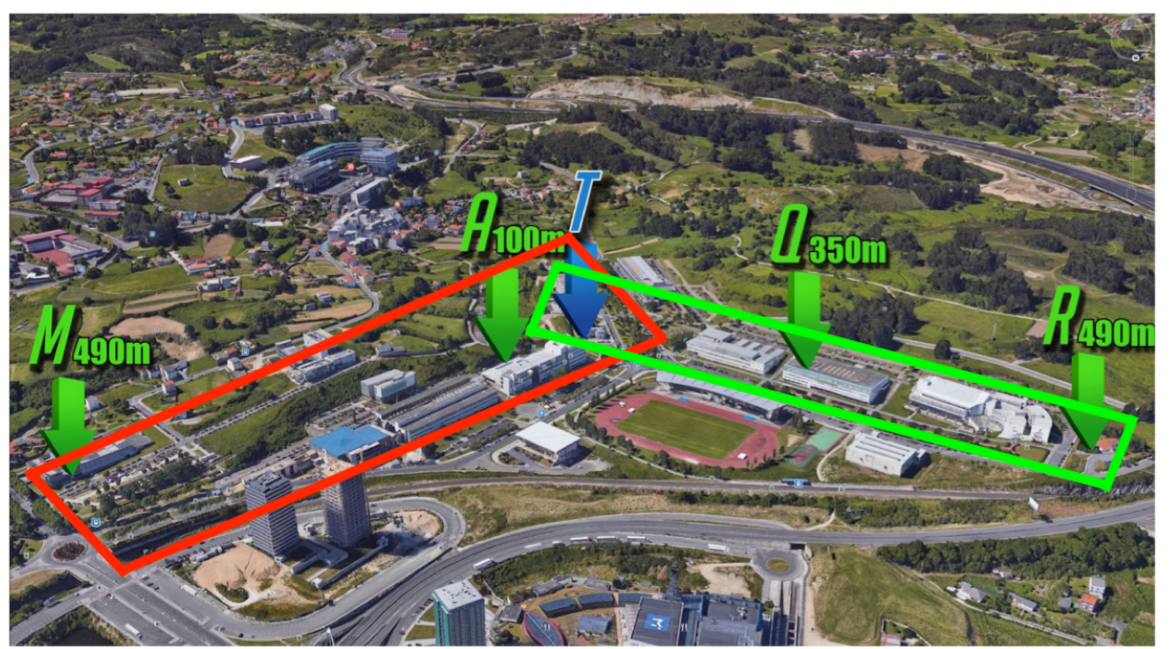

Figure 1. Smart campus modeled with the 3D ray launching tool (source: (c)2018 Google).

\subsection{Planning Simulator}

The in-house-developed 3D Ray Launching (3D-RL) technique is based on Geometrical Optics (GO) and the Uniform Theory of Diffraction (UTD). The first step is the creation of the scenario under analysis considering all the obstacles within it, such as buildings, vehicles, vegetation, or people. This design phase is crucial in order to obtain accurate results for the real environment. Once the 
scenario is created properly, the frequency of operation, number of reflections, radiation pattern of the transceivers, and angular and spatial resolution can be fixed as input parameters in the algorithm for simulation. Then, the whole scenario is divided into a 3D mesh of cuboids, in which all the electromagnetic phenomena are saved during simulation, emulating the electromagnetic propagation of the real waves. A further description of the 3D-RL tool can be found in [8].

Smart campus ecosystems are challenging environments in terms of radio propagation analysis due to their large dimensions, as well as multipath propagation, caused by the multiple obstacles within them. Hence, in order to achieve a good trade-off between simulation computational cost and results' accuracy, it is highly important to consider the optimal parameters for the number of reflections and the angular and spatial resolution of the RL algorithm. Thus, an analysis of the optimal input parameters for the RL tool applied in large complex environments was presented in [9]. Such previous results have been considered to obtain the simulation parameters for the proposed smart campus scenario, which are summarized in Table 1. The scenarios created for the simulations are shown in Figure 2, which correspond to the two scenarios indicated in Figure 1. In order to obtain accurate results, the created scenarios include buildings, sidewalks, roads, trees, and cars, as well as realistic sizes and material properties (permittivity and conductivity). As an example, Figure 3 shows the estimated Radio Frequency (RF) power distribution for a bi-dimensional plane at two different heights when the transmitter is located at a 1.8-m height on the building at the top on the right of the scenario shown in Figure 2a.

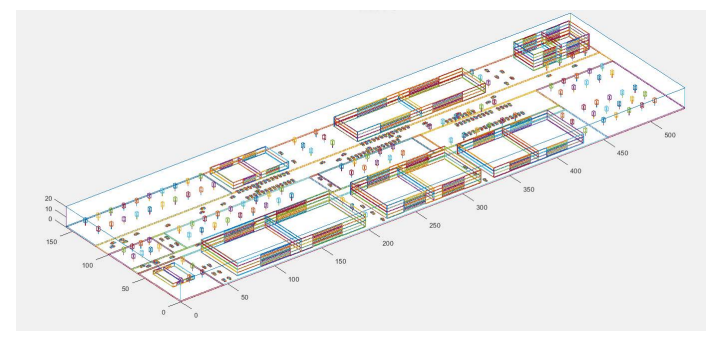

(a)

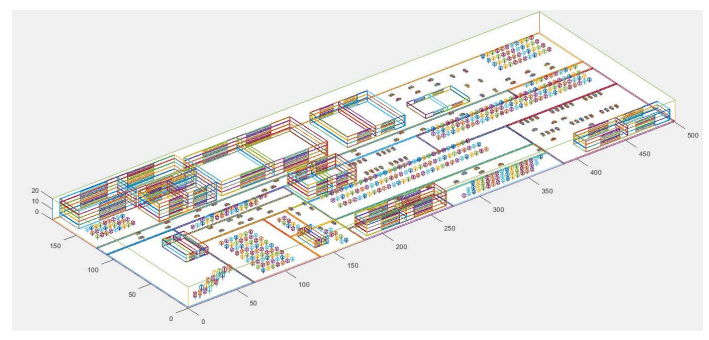

(b)

Figure 2. Simulated scenarios of the smart campus. (a) Red scenario; (b) green scenario.

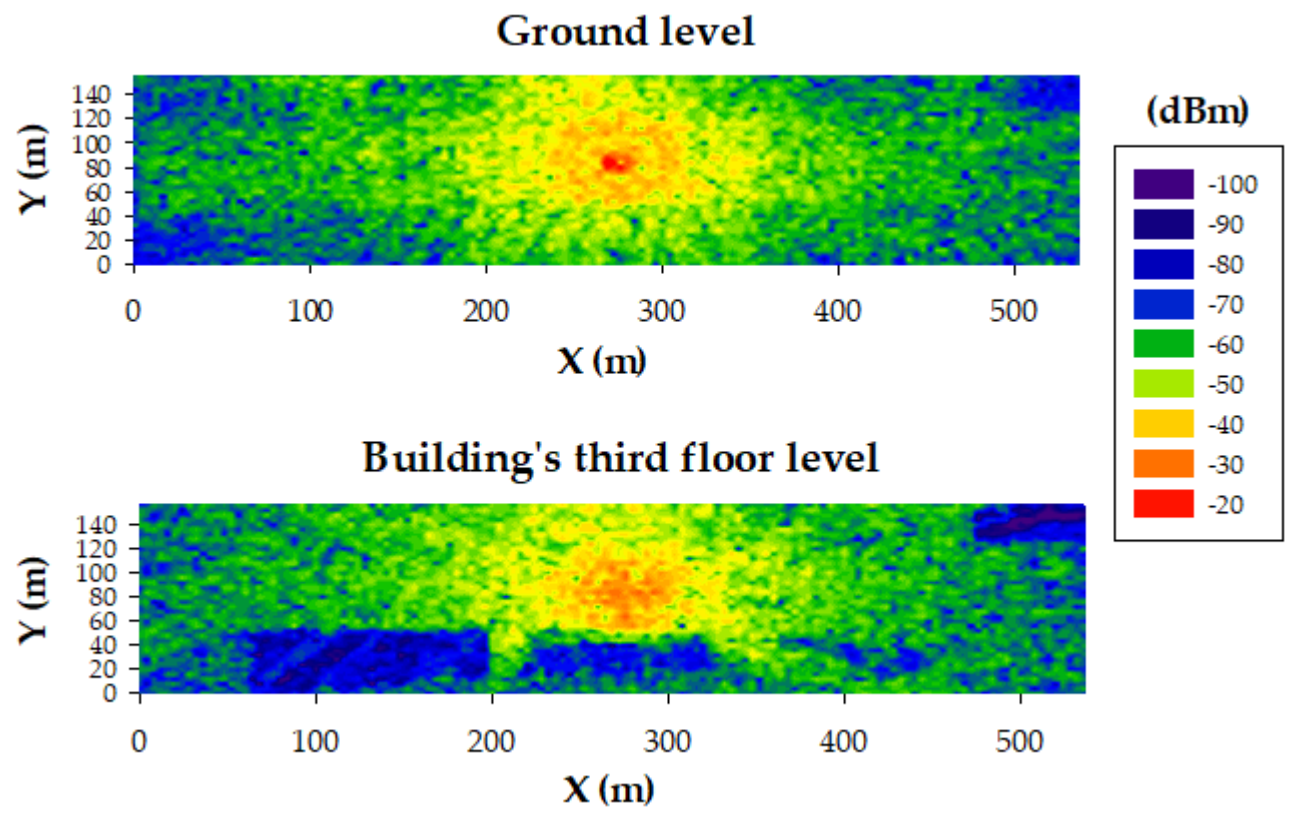

Figure 3. Example of the simulation results of Scenario (Figure 2a): bi-dimensional planes of the estimated RF power distribution for two different heights. 
Table 1. 3D ray launching parameters.

\begin{tabular}{cc}
\hline Parameter & Value \\
\hline Operation frequency & $868.3 \mathrm{MHz}$ \\
Output power level & $14 \mathrm{dBm}$ \\
Permitted reflections & 6 \\
Cuboid resolution & $4 \mathrm{~m} \times 4 \mathrm{~m} \times 4 \mathrm{~m}$ \\
Launched rays' resolution & $1^{\mathrm{o}}$ \\
Antenna type and gain & Monopole, $0 \mathrm{dBi}$ \\
\hline
\end{tabular}

\subsection{Empirical Validation: LoRaWAN Testbed}

In order to evaluate LoRaWAN performance in a real environment, a small LoRaWAN network consisting of a gateway and a node was deployed. A RisingHF RHF0M301 module [10] installed on a Raspberry Pi 3 was selected as gateway, and a RisingHF RHF76-052 module [11] was used as the LoRaWAN node (in Figure 4). The performed tests consisted of transmitting several packages (up to ten packages per location) from the LoRaWAN node to the gateway using acknowledgment messages at different spots on the campus. Figure 5 shows such spots (in red circles) together with the LoRaWAN gateway location (the green circle). The transmitter was placed near a window inside a building, at a height of $3.5 \mathrm{~m}$ from the street's ground level. In contrast, all the measurement spots were located outdoors, at a height of $0.5 \mathrm{~m}$. At all the previously-mentioned spots, the Received Signal Strength Indicator (RSSI) and Signal-to-Noise Ratio (SNR) values were recorded both for the LoRaWAN gateway and the node.

To test for possible interferences in the Industrial, Scientific, and Medical (ISM) sub-band, a USRP B210 [12] with the same 0-dBi antenna used by the LoRaWAN gateway was connected to a laptop that acted both as the data-logger and spectrum analyzer (such a measurement system can be observed in Figure 4). The laptop was configured to monitor a central frequency of $868.3 \mathrm{MHz}$ with a sampling rate of $1 \mathrm{MHz}$.

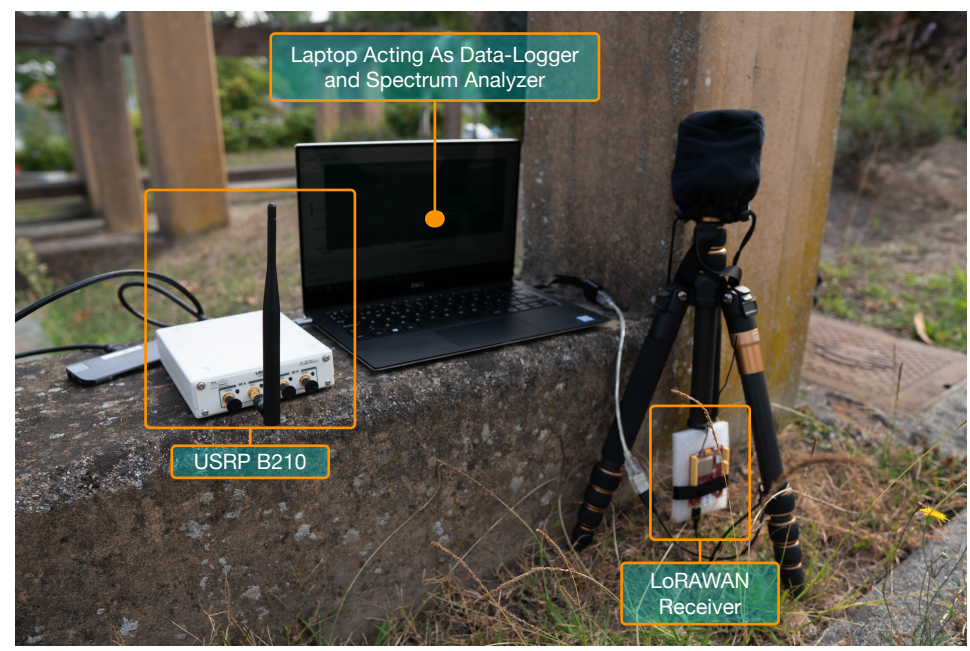

Figure 4. One of the instants during the empirical measurement campaign. 


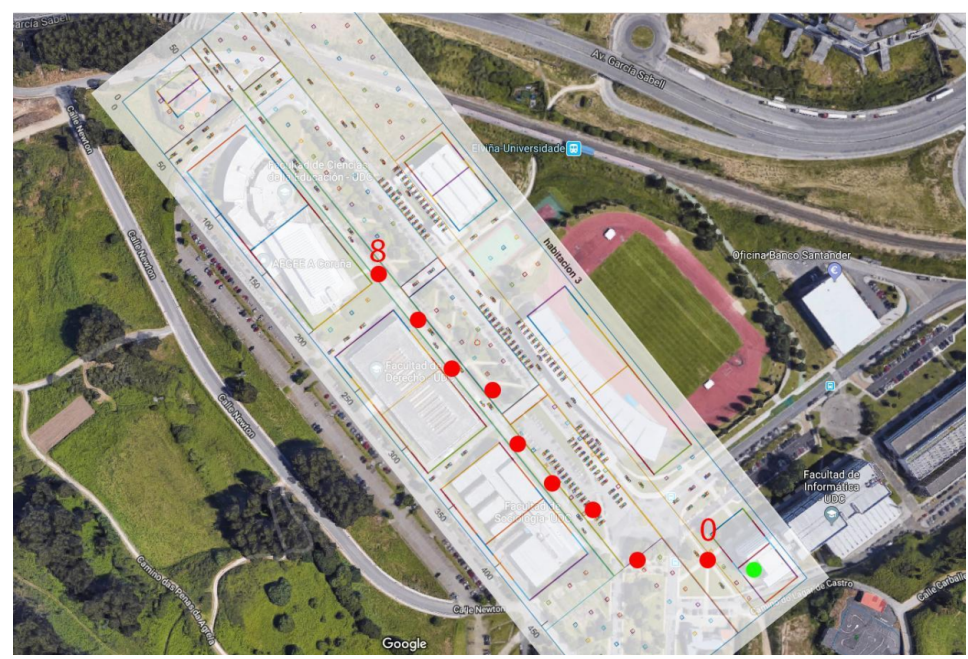

Figure 5. Empirical measurement points.

\subsection{Result Comparison}

The comparison between the measured values and the simulation estimations is depicted in Figure 6. The obtained estimations followed the tendency of the measurement points, and the mean error was only $0.18 \mathrm{~dB}$; however, the standard deviation was $8.07 \mathrm{~dB}$, which was a rather higher value when comparing to the values usually provided by the $3 \mathrm{D}$ ray launching algorithm. These results of the simulation tool may be related to the size of the scenario itself (and the chosen simulation parameters such as cuboid size and launched rays' resolution), since it is the largest scenario simulated so far by the developed 3D ray launching tool. In addition, the fact that measurements were based on RSSI values provided by the motes has to be noted, which inherently added a received RF power level error.

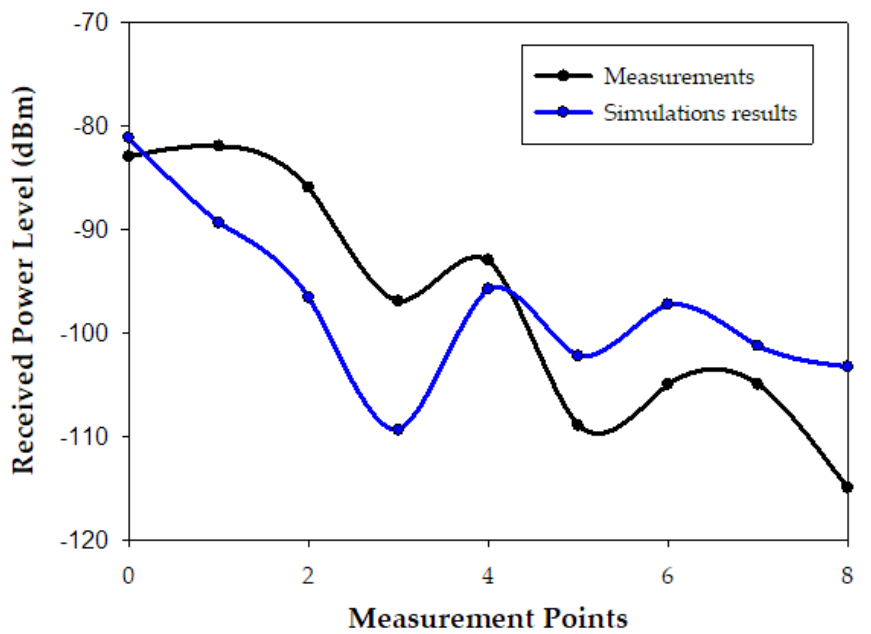

Figure 6. Comparison between measurements and 3D ray launching simulation results.

\section{Conclusions}

This work proposed the design and validation of a LoRaWAN-based smart campus. In order to evaluate the performance of the described system, the received power level obtained by an in-house-developed 3D ray launching planning simulation was compared to empirical LoRaWAN measurements. The preliminary results look promising, but the characteristics of the campus clearly affected the performance, so further work will be devoted to obtain a more accurate model. Therefore, the presented results can provide some guidelines in the network planning phases prior to the deployment of LoRaWAN, specifically for large-scale smart city deployments. 
Author Contributions: T.M.F.-C., P.L.-I., and P.F.-L. conceived of and designed the experiments; T.M.F.-C., and M.S.-A. performed the experiments; M.C.-E., P.L.-I., and L.A. created the scenario and performed the simulations; P.L.-I., E.A., and F.F. processed the simulation results; T.M.F.-C., P.L.-I., and P.F.-L. analyzed the data; T.M.F.-C., P.F.-L., P.L.-I., L.A., F.F., and L.C. wrote the paper.

Acknowledgments: This work has been funded by the Xunta de Galicia (ED431C 2016-045, ED431G/01), the Agencia Estatal de Investigación of Spain (TEC2016-75067-C4-1-R) and ERDF funds of the EU (AEI/FEDER, UE).

Conflicts of Interest: The authors declare no conflict of interest.

\section{References}

1. Hernández-Rojas, D.L.; Fernández-Caramés, T.M.; Fraga-Lamas, P.; Escudero, C.J. Design and Practical Evaluation of a Family of Lightweight Protocols for Heterogeneous Sensing through BLE Beacons in IoT Telemetry Applications. Sensors 2018, 18, 57.

2. Froiz-Míguez, I.; Fernández-Caramés, T. M.; Fraga-Lamas, P.; Castedo, L. Design, Implementation and Practical Evaluation of an IoT Home Automation System for Fog Computing Applications Based on MQTT and ZigBee-WiFi Sensor Nodes. Sensors 2018, 18, 2660.

3. Sanchez-Iborra, R.; Cano, M.-D. State of the Art in LP-WAN Solutions for Industrial IoT Services. Sensors 2016, 16, 708.

4. LoRa-Alliance. LoRaWAN What Is It? A Technical Overview of LoRa and LoRaWAN; White Paper; The LoRa Alliance: San Ramon, CA, USA, 2015.

5. Loriot, M.; Aljer, A.; Shahrour, I. Analysis of the use of LoRaWan technology in a large-scale smart city demonstrator. In Proceedings of the 2017 Sensors Networks Smart and Emerging Technologies (SENSET), Beirut, Lebanon, 12-14 September 2017; pp. 1-4.

6. Wang, S.; Zou, J.; Chen, Y.; Hsu, C.; Cheng, Y.; Chang, C. Long-Term Performance Studies of a LoRaWAN-Based PM2.5 Application on Campus. In Proceedings of the 2018 IEEE 87th Vehicular Technology Conference (VTC Spring), Porto, Portugal, 3-6 June 2018; pp. 1-5.

7. Lee, H.; Ke, K. Monitoring of Large-Area IoT Sensors Using a LoRa Wireless Mesh Network System: Design and Evaluation. IEEE Trans. Instrum. Meas. 2018, 67, 2177-2187.

8. Azpilicueta, L.; Rawat, M.; Rawat, K.; Ghannouchi, F.; Falcone, F. Convergence Analysis in Deterministic 3D Ray Launching Radio Channel Estimation in Complex Environments. ACES J. 2014, 29, 256-271.

9. Azpilicueta, L.; Lopez-Iturri, P.; Aguirre, E.; Vargas-Rosales, C.; León, A.; Falcone, F. Influence of Meshing Adaption in Convergence Performance of Deterministic Ray Launching Estimation in Indoor Scenarios. J. Electromagn. Waves Appl. 2017, 31, 544-559.

10. RHF0M301 LoRaWAN Module Datasheet. Available online: https:/ / www.robotshop.com/media/files / pdf/915mhz-lora-gateway-raspberry-pi-hat-datasheet1.pdf (accessed on 22 October 2018).

11. LoRaWAN Module RHF76-052 Datasheet. Available online: https:/ /fccid.io/2AJUZ76052/User-Manual/ Users-Manual-3211050.pdf (accessed on 22 October 2018).

12. Ettus Research USRP B210. Available online: https://www.ettus.com/product/details/UB210-KIT (accessed on 22 October 2018). 